\title{
Rapid Thermal Sulfurization of Tantalum Film with Enhanced Specific Capacitance
}

\author{
Received: 15.11.2020 \& Accepted: 29.03.2021 \\ HUAZHONG LiU ${ }^{a, b}$, WenXUAN HE ${ }^{b, *}$, \\ XiaOlan WANG ${ }^{a}, Z_{\text {HigaO } \mathrm{LAN}^{a} \text { AND HUOXI XU }}{ }^{a}$ \\ ${ }^{a}$ School of physics and Telecommunications, Huanggang Normal University, Huanggang, China \\ ${ }^{b}$ Department of Basic Courses, Wuhan Donghu University, Wuhan, China
}

Doi: 10.12693/APhysPolA.139.642

*e-mail: WenxuanHe68@hotmail.com

\begin{abstract}
In this paper, the effect of rapid thermal sulfurization on the electrochemical storage properties of ultrathin metallic tantalum ( $\mathrm{Ta}$ ) is reported. It was achieved by introducing sulfur (S) onto the surface and subsurface region of the Ta film, which resulted in areal capacitance enhancement as compared to that of the pristine Ta film. Notably, the sulfurized Ta-based electrode delivers an areal capacitance reaching $3.4 \mathrm{mF} / \mathrm{cm}^{2}$ at scan rate of $5 \mathrm{mV} / \mathrm{s}$ (equivalent to volumetric capacitance of $261.5 \mathrm{~F} / \mathrm{cm}^{3}$ ), in $1 \mathrm{M} \mathrm{Na}_{2} \mathrm{SO}_{4}$ electrolyte solution, with a cycling stability of $76 \%$ over 10,000 consecutive cycles). Surface chemistry and morphology of the electrodes before and after sulfurization were investigated and correlated with the electrochemical properties of different electrodes. The present results anticipate tantalum sulfide $(\mathrm{TaS})$ to be a promising material for electrochemical capacitors.
\end{abstract}

topics: thermal sulfurization, electrochemical capacitors, tantalum

\section{Introduction}

Pseudo-capacitive materials which store charges by surface faradaic reactions often show high power density and long operation lifetimes, when compared to lithium-ions batteries [1, 2]. Among the most studied pseudo-capacitive materials, one can mention transition metal oxides such as $\mathrm{CoO}[3]$ and $\mathrm{NiO}$ [4], conducting polymers [5], and transition metal nitrides including VN [6, 7], TiN [8,9] and MoN [10]. Recently, transition metal chalcogenides (TMCs) have drawn tremendous research interest for energy conservation as pseudo-capacitive materials [11, 12]. Among them, MoS [13], VS [14] and TiS [15] have been proposed as active materials for electrochemical capacitors (ECs) with exceptional performance. In contrast to metal nitride, the screening of TMDs in literature for EC application is still limited. For instance, the use of $\mathrm{TaS}_{2}$ as an electrochemical capacitor material is poorly reported. As one of the typical TMDs, $\mathrm{TaS}_{2}$ exhibits a combination of good chemical, mechanical, and electronic properties, which have been studied in the past [16-21].

Indeed, its structure and electronic properties were reported to be promising for high-power device applications [20,22]. Thus, its properties can also be beneficial in the case of energy storage application. In view of literature on the use of $\mathrm{TaS}_{2}$ for ECs, Wu et al. [23] have reported a $\mathrm{TaS}_{2}$ monolayer with high volumetric capacitance, while Sun et al. [24] have reported ultra-long hybrid microfibers from nanosheets of reduced graphene oxide and $\mathrm{TaS}_{2}$ for super-capacitor application.

In this work, a rapid thermal sulfurization of an ultrathin Ta film at 400 and $600^{\circ} \mathrm{C}$ is reported.

The incorporation of sulfur was revealed by an X-ray photoelectron spectroscopy. In addition, the specific capacitance of the sulfurized electrode was evaluated in $1 \mathrm{M} \mathrm{Na}_{2} \mathrm{SO}_{4}$ and the effect of sulfur incorporation was highlighted and correlated with the electrochemical response of the electrodes.

\section{Experimental}

\subsection{Ta film deposition}

Tantalum ultra-thin films (Ta) were deposited on (110) silicon substrates by DC magnetron sputtering of tantalum targets (purity 99.9\%, 2 inches in diameter) in a sputter machine equipped with a turbo molecular pump that allows achieving a base pressure of $5 \times 10^{-4}$ mbar. The reactive sputtering was carried out without substrate heating. The argon flux was kept constant at $30 \mathrm{sccm}$. The deposition time was fixed to $10 \mathrm{~min}$ at constant pressure of $5 \times 10^{-3} \mathrm{mbar}$. The applied power on a Ta target was $50 \mathrm{~W}$. The obtained thickness of the films as measured for the cross-sectional SEM images was around $130 \mathrm{~nm}$. 


\subsection{Rapid thermal sulfurization of Ta films}

The rapid thermal sulfurization process was employed to introduce sulfur to the as-deposited Ta films (using OTF-1200X setup). The sulfurization was performed on a quartz tube (reactor). Firstly, the Ta film was introduced into the reactor with the $\mathrm{S}$ powder $(0.3 \mathrm{~g})$, and this was placed $10 \mathrm{~cm}$ away from the Ta film. The S powder (sigma Aldrich, $99.98 \%$ ) was used without any further purification. Secondly, the reactor was flushed for one hour to remove (reduce) oxygen, using a $0.500 \mathrm{~L} / \mathrm{min}$ argon flow outside of the furnace, prior to the sulfurization process. After that, the reactor was put inside of the furnace. Finally, under nitrogen flux the furnace was heated to two different temperatures in each case. For this study, temperatures of 400 or $600{ }^{\circ} \mathrm{C}$, under nitrogen pressure of 40 Torr and 30 min duration, were chosen. After the reaction, the $\mathrm{N}_{2}$ flow was stopped, and the quartz tube was removed from the reactor and was cooled with the argon flow for $1 \mathrm{~h}$.

It was noticed that temperatures of $\geq 650{ }^{\circ} \mathrm{C}$ led to film delamination and cracking. The asdeposited and the treated Ta films at 400 and $600{ }^{\circ} \mathrm{C}$ are assigned as Ta, Ta-400 and Ta-600, respectively. Such rapid thermal sulfurization aims at improving the capacitive properties, as it will be shown in the next section.

\subsection{Film characterization}

The film thickness values were determined through both SEM (using scanning electron microscopy (SEM) (JSM7600F; JEOL) examination of the film cross-section and average step height measurement using a stylus profilometer (Dektak; Bruker, Germany). The phase composition of the samples was investigated by means of X-ray diffraction (XRD) studies using a diffractometer (PANalytical Empyrean; Malvern Panalytical Ltd, $\mathrm{UK})$ operating with monochromatic $\mathrm{CuK}_{\alpha}$ radiation $(\lambda=0.1546 \mathrm{~nm})$ in the grazing incidence configuration (grazing angle, $\mathrm{GA}=0.5^{\circ}$ ) at a voltage of $45 \mathrm{kV}$ and a current of $40 \mathrm{~mA}$. The microstructural characterization was carried out by a field emission gun transmission electron microscope using a Hitachi HNAR 9000 microscope operating at $300 \mathrm{kV}$.

The microstructural and surface morphology characterizations of the samples were examined by atomic force microscopy (AFM) in contact mode (NanoWizard II BioAFM). For surface chemical analysis, X-ray photoelectron spectroscopy (XPS) measurements were carried out on a Kratos Axis Ultra spectrometer using a monochromatic $\mathrm{AlK}_{\alpha}$ radiation $(1486.68 \mathrm{eV})$ with a spot size of $250 \times 250 \mu \mathrm{m}^{2}$. A flood gun was used for charge compensation and the $\mathrm{C} 1 \mathrm{~s}$ line at $284.5 \mathrm{eV}$ was considered as a reference for the binding energies correction shift.
The XPS depth profiles were built by successively alternating $100 \mathrm{~s}$ sputtering with $1 \mathrm{keV} \mathrm{Ar}{ }^{+}$beam and XPS analysis performed with a monochromatic $\mathrm{AlK}_{\alpha}$ X-ray beam with a spot size of $300 \mu \mathrm{m}$. Quantification was evaluated after Shirley background subtraction with Avantage software on survey spectra acquired at $150 \mathrm{eV}$ pass energy.

\subsection{Electrochemical characterization}

Electrochemical studies of the Ta electrodes before and after sulfurization were carried out using a potentiostat/galvanostat PGSTAT128N at room temperature in a typical three-electrode electrochemical cell. Ta samples, $\mathrm{Ag} / \mathrm{AgCl}$ and platinum foil were used as working, reference and counter electrodes, respectively. In this configuration, only the Ta-coated side was in contact with the electrolyte $\left(\mathrm{S}=0.5 \mathrm{~cm}^{2}\right)$, while the other side was in contact with a current collector through conductive silver paste to ensure good contact. Cyclic voltammetry (CV) and galvanostatic charge-discharge (GCD) experiments were performed in $1.0 \mathrm{M} \mathrm{Na}_{2} \mathrm{SO}_{4}$ aqueous solution. Capacitance $C$ of the samples values were calculated from $\mathrm{CV}$ and GCD data using

$$
C=\frac{\Delta_{i}}{2 v}
$$

where $\Delta_{i}$ and $v$ are the respective values of average of the intensity difference between upper and lower parts of the CV curve, and the scan rate, respectively.

It is worth to mention that only the areal capacitance $\left[\mathrm{mF} / \mathrm{cm}^{2}\right]$ is evaluated in this work. Indeed, due to the small material quantity deposited in the form of ultrathin film ( $\sim 140 \mathrm{~nm}$ thick $)$ the mass of the Ta deposit is hard to estimate, and thus the gravimetric capacitance $[\mathrm{F} / \mathrm{g}]$ cannot be estimated. The latter is mostly reported in the case of powders. Furthermore, in the case of thin films, the most important presentation of the specific capacitance is the areal capacitance for practical applications.

\section{Results and discussion}

The 3D AFM images of Ta electrodes before and after thermal sulfurization at 400 and $600{ }^{\circ} \mathrm{C}$ are shown in Fig. 1a-c, respectively. The AFM images demonstrate the presence of numerous features in the form of hills and duns on the surface. The surface roughness increases from 19.8 to 32.4 and $143.0 \mathrm{~nm}$ for Ta, Ta-400 and Ta-600 electrodes, respectively. This indicates that thermal sulfurization leads to the increase of surface roughness and thus the area at the surface region. Such surface modification is anticipated to enhance the electrode capacitance.

The XRD diagrams (Fig. 2) on these samples show one sharp crystalline peak, centered at $2 \theta \approx 38^{\circ}$ corresponding to the crystalline nature of Ta. The peak observed at $2 \theta \approx 38^{\circ}$ can 


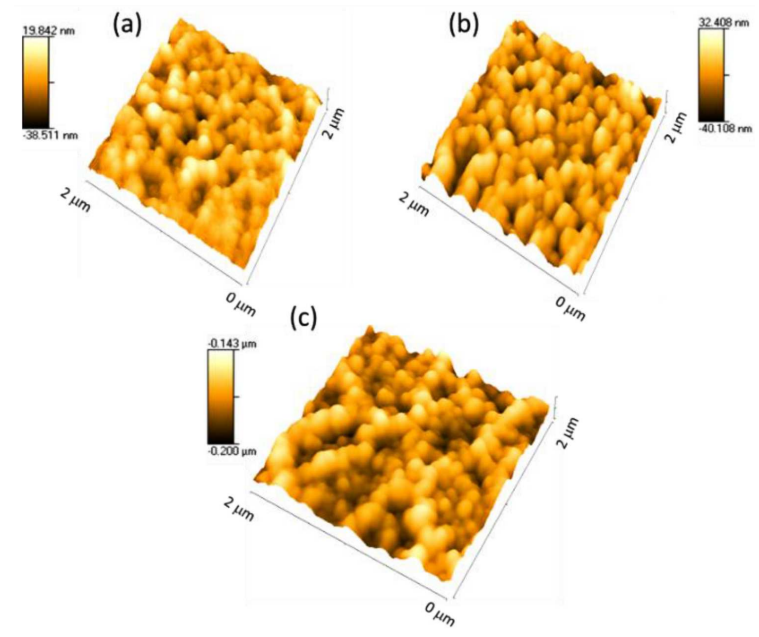

Fig. 1. AFM 3D-image of (a) Ta, (b) Ta-400 and (c) Ta-600 electrodes.

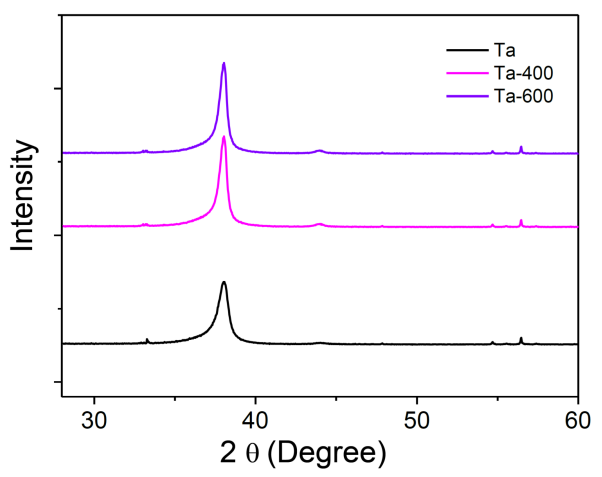

Fig. 2. XRD patterns of Ta electrodes before and after thermal sulfurization.

be ascribed to either (110) of bcc-Ta or (200) of $\beta$-Ta [25-27]. The thermal sulfurization leads to a peak shift toward high angles by $0.05^{\circ}$, which can be due to stress relaxation. No additional peak related to $\mathrm{Ta}-\mathrm{S}$ was observed, which indicates that the sulfur incorporation could take place only at the surface region.

Because of its surface sensitivity, XPS is a powerful method for the investigation of surface composition changes due to the thermal sulfurization of Ta electrodes. The XPS survey spectra, shown in Fig. 3 of the as-deposited and treated Ta films confirmed the presence of tantalum $(\mathrm{Ta})$, oxygen $(\mathrm{O})$, carbon (C) on the surface with small peak of sulfur (S) in the case of treated electrodes. The XPS peak due to carbon in all the films may be attributed to the surface contamination in the form of adsorbed carbon species. In addition, the presence of oxygen can be attributed to oxygen incorporation on the surface during synthesis and/or aging.

Table I lists the elemental composition of the three Ta film surfaces before and after the RTA treatment. As can be noticed, the S percentage increases from 1.7 to 3.6 at.\%, for Ta-400 and T-600,

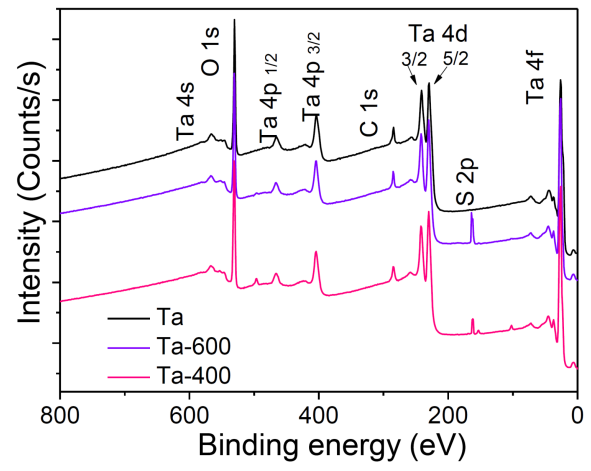

Fig. 3. XPS survey spectra of Ta electrodes before and after thermal sulfurization.

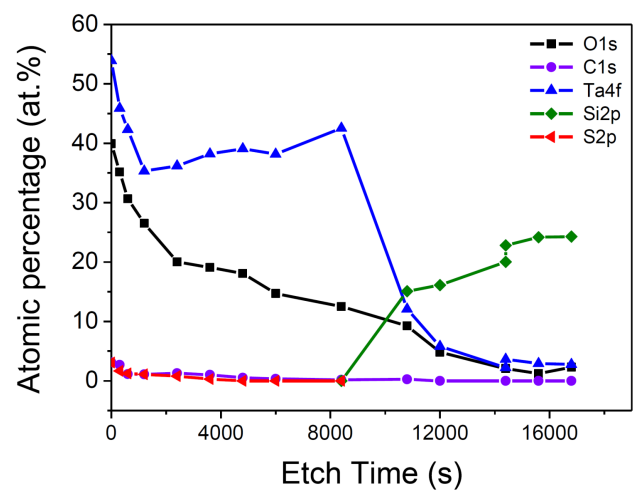

Fig. 4. XPS depth profiles of O, C, Ta, S and Si elements for Ta-600 electrode.

TABLE I

XPS analysis of the Ta films surfaces before and after thermal sulfurization giving elemental composition.

\begin{tabular}{c|c|c|c}
\hline \hline & Ta & Ta-400 & Ta-600 \\
\hline Ta $4 f$ [at. \%] & 59.7 & 51.8 & 51.4 \\
O 1s [at. \%] & 37.1 & 42.1 & 41.2 \\
C 1s [at. \%] & 3.2 & 4.4 & 3.8 \\
S 2p [at. \%] & - & 1.7 & 3.6 \\
N 1s [at. \%] & - & - & -
\end{tabular}

respectively. This indicates that the increase of annealing temperature leads to more sulfur incorporation on the surface of the electrodes.

In order to probe the incorporation of $\mathrm{S}$ in the treated films, we have performed XPS depth profiling of the Ta-600 film as shown in Fig. 4. This kind of profiling permits a clear visualisation of the elemental distribution from the film surface to the bulk region. The $\mathrm{Ta}$ and $\mathrm{O}$ are both accumulated on the surface (without etching, $t=0$ ). The $\mathrm{O}$ decreases simultaneously with etching time increase from the top surface to the bulk, while the Ta percentage amount increases with the etching time increase. This indicates surface oxidation of the film since the Ta and $\mathrm{O}$ behave oppositely. Concerning the $\mathrm{S}$, its amount also decreases with the etching time increase. However, the value tends to almost zero 

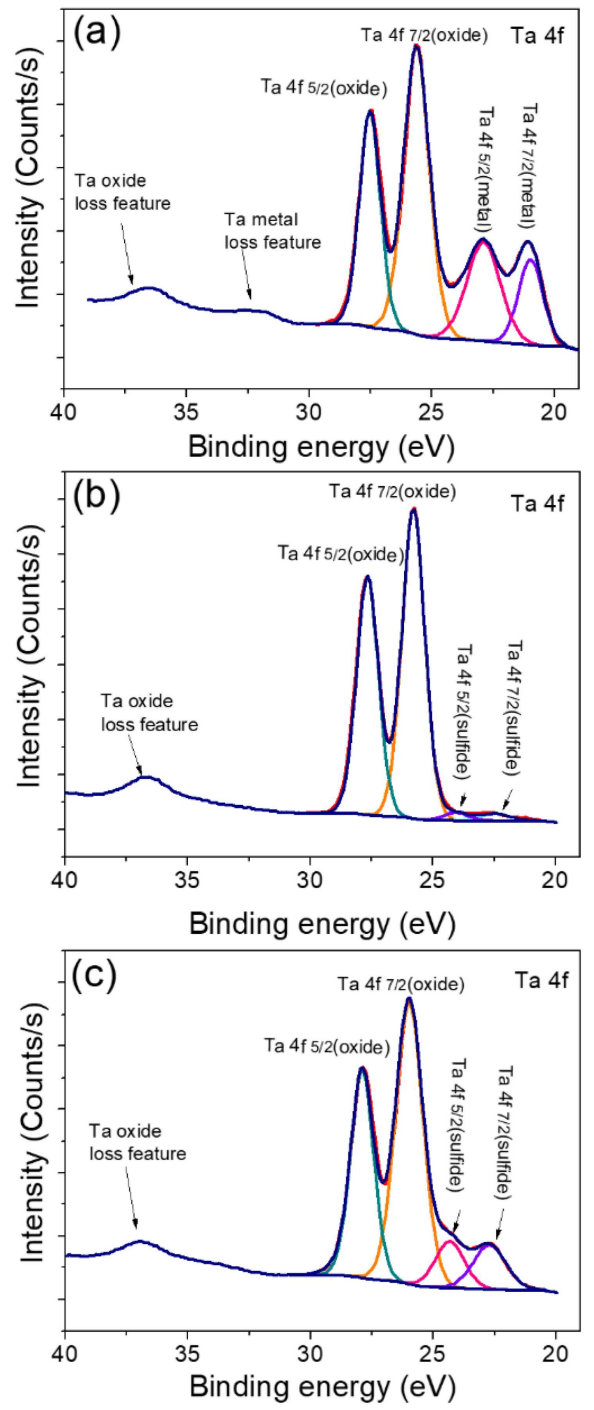

Fig. 5. Ta $4 f$ high-resolution XPS spectra for the (a) as-deposited Ta, (b) Ta-400 and (c) Ta-600 films.

before that of $\mathrm{O}$ and $\mathrm{Ta}$, which indicates that $\mathrm{S}$ accumulates only at the surface and subsurface region of the film. Indeed, this can be enough to change the electrochemical properties of the film since ECs is a surface region process.

The Ta $4 f$ XPS high-resolution spectra of the Ta, Ta-400 and Ta-600 electrode surfaces are shown in Fig. 5a-c, respectively. In the case of the $\mathrm{Ta}$ film, the peak deconvolution revealed that several chemical states contributed to the overall spectrum. A doublet of $\mathrm{Ta} 4 f 7 / 2$ and $\mathrm{Ta} 4 f 5 / 2$ located at about 21.2 and $23.1 \mathrm{eV}$, respectively, can be attributed to metallic tantalum [28, 29]. In addition, another pair of peaks at approximately 25.6 and $27.5 \mathrm{eV}$ can be ascribed to Ta $4 f 7 / 2$ and Ta $4 f 5 / 2$, respectively, which can be assigned to tantalum oxide [28-30]. In the case of the Ta-400 (Fig. 5b) and Ta-600 (Fig. 5c) films, the pair of peaks that are attributed to tantalum oxide, remains always presents in both films. However, the pair that was attributed
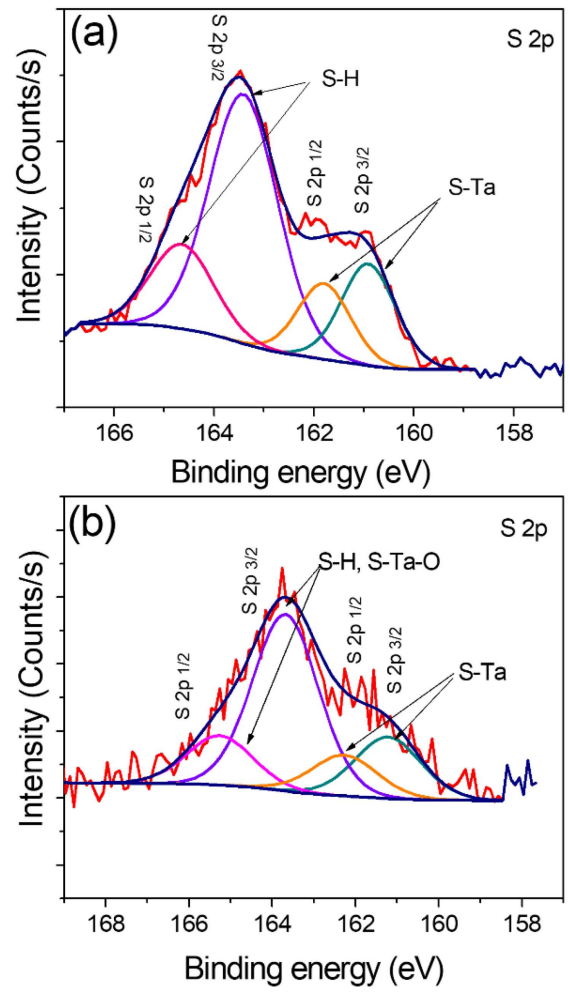

Fig. 6. S $2 p$ high-resolution XPS spectra for (a) Ta-400 and (b) Ta-600 films.

to metallic tantalum disappears and two new peaks centered at 22.7 and $24.3 \mathrm{eV}$ appear in the case of sulfurized films. These peaks are attributed to Ta $4 f 7 / 2$ and Ta $4 f 5 / 2$, respectively, in tantalum sulfide (TaS) [30, 31].

It is worth to mention that the intensity of $\mathrm{Ta}-\mathrm{S}$ peaks in Ta-600 film is higher that those in Ta-400 films which is consistent with the values of sulfur reported in Table I, since Ta-600 contains more sulfur than the Ta-400 film.

The S $2 p$ spectra of the treated Ta-400 and Ta-600 films were also fitted to develop an insight of any surface chemistry changes as a result of thermal sulfurization (Fig. 6). In both films, the S element can be deconvoluted into two independent components centered at 160.9 and $163.3 \mathrm{eV}$, which correspond to S-Ta (S $2 p 3 / 2)$ and/or S-O-Ta $(\mathrm{S} 2 p 3 / 2) / \mathrm{S}-\mathrm{H}$ (S $2 p 3 / 2$ ) bonds, respectively $[23,31,32]$. Nonetheless, it is believed that the peaks at $163.3 \mathrm{eV}$ are more probably attributed to $\mathrm{S}-\mathrm{O}-\mathrm{Ta}(\mathrm{S} 2 \mathrm{p} 3 / 2)$ due to the high oxygen content on the film surfaces. The $\mathrm{S} 2 p$ and Ta $4 f$ peak deconvolutions indicate an increased number of surface defects such as undercoordinated Ta atoms, and oxidized Ta [23] due to sulfur insertion.

Figure 7 presents the cyclic voltammograms (CV) of the as-deposited and sulfur-treated Ta electrodes in $1 \mathrm{M} \mathrm{Na} \mathrm{NO}_{4}$ electrolyte solution at different scan rates (5 to $1000 \mathrm{mV} / \mathrm{s}$ ) in a potential window of $(-0.8-0)$. The as-deposited Ta metallic electrode exhibits low capacitive current density 

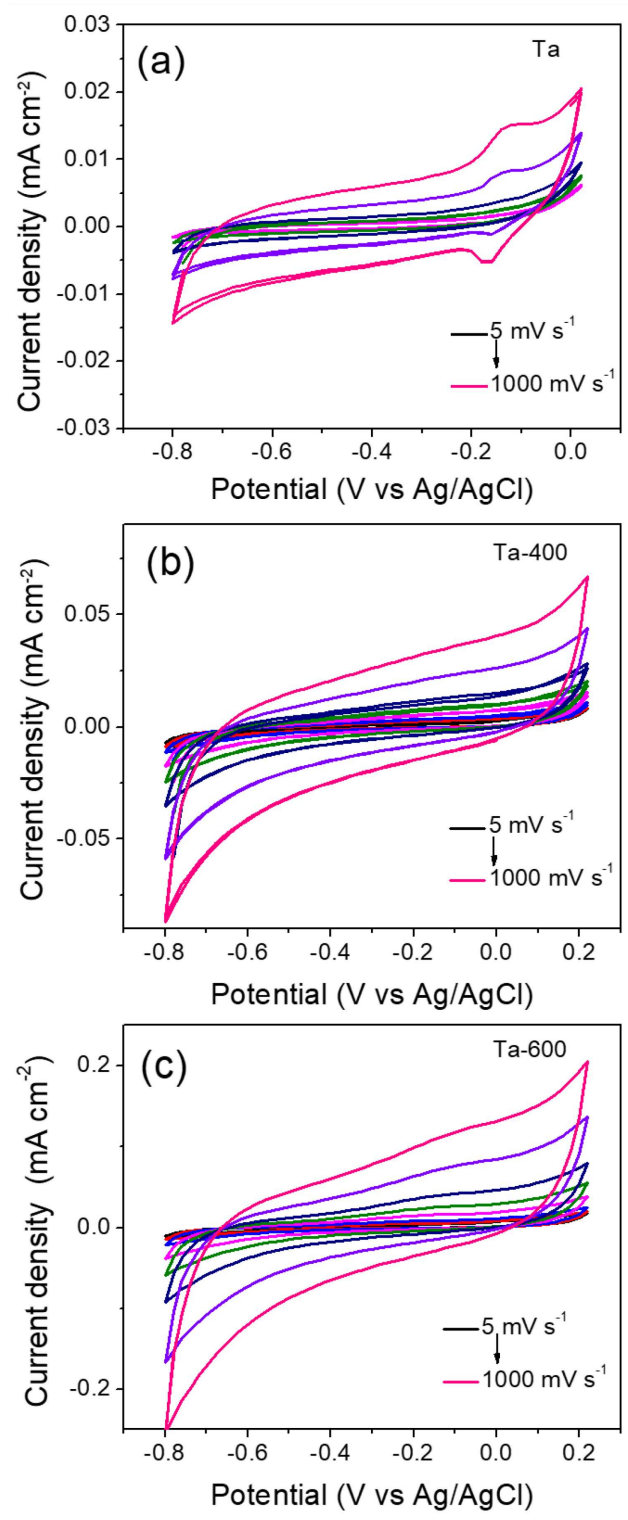

Fig. 7. $C V$ curves at various scan rates of (a) asdeposited Ta, (b) Ta-400, and (c) Ta-600 electrodes.

with a oxidation/reduction peaks (may be due to metal oxidation at this potential window) at around $-0.27 \mathrm{eV}$ (Fig. 7a). Upon RTA treatment at $400^{\circ} \mathrm{C}$ and $600{ }^{\circ} \mathrm{C}$, the $\mathrm{CV}$ curves exhibited a capacitive current, but without any oxidation/reduction peaks. The CV curves of Ta-400 and Ta-600 (Fig. 7b and c) revealed a near-symmetric rectangular shape even at a high scan rate of $1000 \mathrm{mV} / \mathrm{s}$, indicative of a quasi-ideal capacitive behavior.

The comparison of the CVs representing the capacitive current of the three electrodes at the scan rate of $100 \mathrm{mV} / \mathrm{s}$ are displayed in Fig. 8a. It is evident that the thermal sulfurization treatment of the Ta electrode at 400 and $600{ }^{\circ} \mathrm{C}$ leads to a noticeable increase in capacitive current (specific capacitance) through a modification of the as-deposited Ta surface into a more electrochemically active electrode. Rapid thermal sulfurization
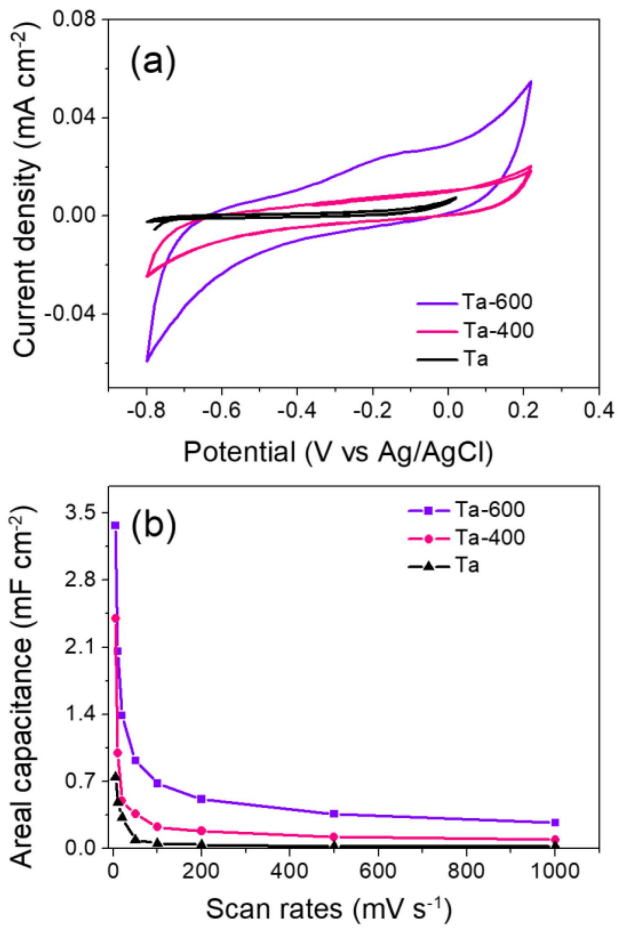

Fig. 8. (a) Comparison of CV curves of Ta based electrodes before and after sulfurization treatment at scan rate of $100 \mathrm{mV} / \mathrm{s}$, (b) variation of areal capacitance of the Ta based electrodes at different scan rates.

annealing may, therefore, be envisioned as a promising route towards producing high energy and power density TMDs electrodes for electrochemical capacitors. The variation in the areal capacitances of the different Ta electrodes before and after thermal sulfurization, as a function of scan rates, are shown in Fig. 8b. It can be seen that the capacitance decreases upon increasing the scan rate, suggesting that the electrode surface becomes partially inaccessible at high scan and charging-discharging rates. Furthermore, it is clear that the Ta-600 electrode has the highest capacitance. Such behavior may be related to the larger surface area of the Ta- 600 film (as demonstrated from AFM analyses) as well as to the presence of a considerable sulfur amount on the surface, when compared with other Ta- and Ta-400-based electrodes. For the Ta-600 electrode, the areal capacitance values calculated from the $\mathrm{CV}$ curves (Fig. 7) using (1) were 3.3 and $0.27 \mathrm{mF} / \mathrm{cm}^{2}$ for 5 and at $1000 \mathrm{mV} / \mathrm{s}$ scan rates, respectively (when compared to 2.4 and $0.09 \mathrm{mF} / \mathrm{cm}^{2}$ for the Ta-400 electrode). Therefore, the areal capacitance was enhanced by 3.4 and 4.5 in the case of the Ta400 and Ta-600, electrodes, respectively, when compared to the as-deposited Ta electrode $\left(0.7 \mathrm{mF} / \mathrm{cm}^{2}\right.$ at $5 \mathrm{mV} / \mathrm{s})$. Taking into account the ultra-thin nature of the deposited films (140 nm thick). Increasing film thicknesses is expected to lead to a considerable increase of the film areal capacitance as already reported in the case of supercapacitor electrodes in the form of films $[6,7]$. 


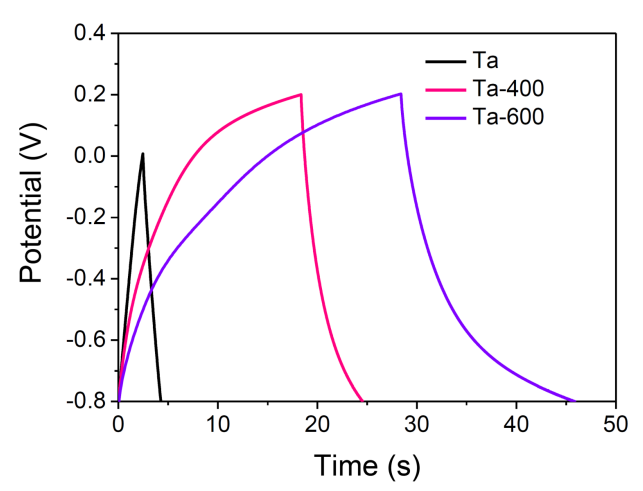

Fig. 9. Galvanostatic charge-discharge curves of (a) Ta-400 and (b) Ta-600 at current density of $0.02 \mathrm{~mA} / \mathrm{cm}^{2}$.

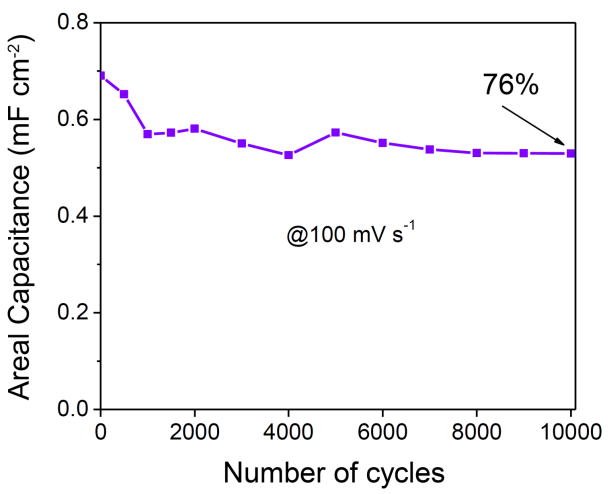

Fig. 10. Evolution of the capacitance during 10,000 consecutive cycles at scan rate of $100 \mathrm{mV} / \mathrm{s}$.

Galvanostatic charge-discharge (GCD) curves at current densities of $0.02 \mathrm{~mA} / \mathrm{cm}^{2}$ for the Ta, Ta- 400 and Ta-600 electrodes are presented in Fig. 9, which displays a voltage drop (IR drop) of $0.061,0.008$ and $0.005 \mathrm{~V}$, respectively. These values attest to the superior electrical conductivity of the Ta-600 electrode, which is consistent with the $\mathrm{CV}$ results.

The capacity loss of the Ta-600 electrode after 10,000 consecutive cycles at a scan rate of $100 \mathrm{mV} / \mathrm{s}$ is $24 \%$, as shown in Fig. 10. This cycling stability is comparable to other TMDs nanostructures with high specific capacitance $[23,24]$.

\section{Conclusions}

In this work, an ultrathin metallic Ta film was deposited by DC-sputtering onto Si substrates, then subjected to rapid thermal sulfurization under nitrogen atmosphere at 400 or $600^{\circ} \mathrm{C}$. The sulfurized films were tested as electrodes in electrochemical capacitors in $1 \mathrm{M} \mathrm{Na}_{2} \mathrm{SO}_{4}$ aqueous electrolyte. It was found that the sulfurization treatment leads to structural and surface chemistry modification of the annealed Ta electrodes, which led to an improvement of their electrochemical storage performance. A 4.5 fold enhancement of the specific capacitance was measured for the electrodes annealed at $600{ }^{\circ} \mathrm{C}$, which was associated with the increase of the surface area, and the presence of the $\mathrm{TaS}_{2}$ phase at the surface and subsurface region. In addition, the capacitance seems to increase with the increase of the sulfur content on the treated Ta surface. The sulfurized electrodes exhibit a maximum areal capacitance of $3.4 \mathrm{mF} / \mathrm{cm}^{2}$ at $5 \mathrm{mV} / \mathrm{s}$ (equivalent to $261.5 \mathrm{~F} / \mathrm{cm}^{3}$ ) which is expected to increase further as the thickness of the Ta deposit increases. In addition, the cycling stability reached 10,000 cycles in $1.0 \mathrm{M} \mathrm{Na}_{2} \mathrm{SO}_{4}$ electrolyte with $76 \%$ capacitance retention. These findings highlight thermal sulfurization as a promising route to synthesis of different transition metal chalcogenides in general and $\mathrm{TaS}$ in particular with improved charge storage properties.

\section{Acknowledgments}

The research was financially supported by the Research Project of Hubei Natural Science Foundation, China (Project No. 2018CFC876).

\section{References}

[1] Z. Stević, M. Rajčić-Vujasinović, S. Bugarinović, A. Dekanski, Acta Phys. Pol. A 117, 228 (2010).

[2] A. Despotuli, A. Andreeva, Acta Phys. Pol. A 120, 260 (2011).

[3] V. Yagmur, F.E. Atalay, H. Kaya, D. Avcu, E. Aydogmus, Acta Phys. Pol. A 123, 260 (2013).

[4] Z. Kavaliauskas, L. Marcinauskas, P. Valatkevicius, Acta Phys. Pol. A 119, 253 (2011).

[5] A. Rudajevová, M. Varga, J. Prokes, J. Kopecká, J. Stejskal, Acta Phys. Pol. A 128, 730 (2015).

[6] A. Achour, R. Lucio-Porto, S. Solaymani, M. Islam, I. Ahmad, T. Brousse, J. Mater. Sci.: Mater. Electron 29, 13125 (2018).

[7] A. Achour, M. Islam, I. Ahmad, K. Saeed, S. Solaymani, Coating 9, 1 (2019).

[8] A. Achour, M. Islam, I. Ahmad, L. Le Brizoual, A. Djouadi, T. Brousse, Scrip. Mater 153, 59 (2018).

[9] A. Achour, M. Chaker, H. Achour et al., J. Power Sources 359, 349 (2017).

[10] Y. Yuan, X.-R. Li, X. Zhang, J. Wang, Y. Zhou, C.-T. Lin, T.-W. Lin et al., Nano Futures 2, 045001 (2018).

[11] L. Lin, W. Lei, S. Zhang, Y. Liu, G.G. Wallace, J. Chen, Energy Stor. Mater. 19, 408 (2019).

[12] M.A. Bissett, S.D. Worrall, I.A. Kinloch, R.A.W. Dryfe, Electrochim. Acta 201, 30 (2016). 
[13] S.S. Karade, D.P. Dubal, B.R. Sankapal, RSC Adv. 6, 39159 (2016).

[14] N.A. Kalam, C. Sengottaiyan, R. Jayavel, K. Ariga, R.G. Shrestha, T. Subramani, S. Sankar et al., J. Taiwan Inst. Chem. Eng. 92, 72 (2018).

[15] X. Zang, C. Shen, E. Kao, R. Warren, R. Zhang, K.S. The, J. Zhong et al., Adv. Mater. 30, 1704754 (2018).

[16] R. Ang, Y. Tanaka, E. Ieki, K. Nakayama, T. Sato, L.J. Li, W.J. Lu et al., Phys. Rev. Lett. 109, 176403 (2012).

[17] B. Sipos, A.F. Kusmartseva, A. Akrap, H. Berger, L. Forro, E. Tutis, Nat. Mater. 7, 960 (2008).

[18] J. Zheng, H. Zhang, S. Dong, Y. Liu, C.T. Nai, H.S. Shin, H.Y. Jeong et al., Nat. Commun. 5, 2995 (2014).

[19] A. W. Tsen, R. Hovden, D. Wang et al., PNAS 112, 15054 (2015).

[20] L.Wang, M.G. Kanatzidis, Chem. Mater 13, 3717 (2001).

[21] L. Ma, C. Ye, Y. Yu, X. F. Lu, X. Niu, S. Kim, D. Feng et al., Nat. Commun 7, 10956 (2016).

[22] M. Yoshida, Y. Zhang, J. Ye, R. Suzuki, Y. Imai, S. Kimura, A. Fujiwara et al., Sci. Rep. 4, 7302 (2014).

[23] J. Wu, J. Peng, Z. Yu, Y. Zhou, Y. Guo, Z. Li, Y. Lin et al., J. Am. Chem. Soc. 140, 493 (2018).
[24] G. Sun, J. Liu, X. Zhang, X. Wang, H. Li, Y. Yu, W. Huang et al., Angew. Chem. Int Ed Engl. 53, 12576 (2014).

[25] S. Shiri, A. Odeshi, N. Chen, R. Feng, R. Sutarto, Q. Yang, Surf. Coat. Tech 358, 942 (2019).

[26] H. B. Nie, S.Y. Xu, S.J. Wang, L.P. You, Z. Yang, C.K. Ong, J. Li et al., Appl. Phys. A 73, 229 (2001).

[27] D.W. Face, D.E. Prober, J. Vac. Sci. Technol. A 5, 3408 (1987).

[28] P. Buabthong, N.B. Stasiewicz, S. Mitrovic, N.S. Lewis, Surf. Sci. Spectra 24, 024001 (2017).

[29] R. Simpson, R.G.White, J.F. Watts, M.A. Baker, Appl. Surf. Sci. 405, 79 (2017).

[30] J. Luxa, V. Mazanek, M. Pumera, P. Lazar, D. Sedmidubsky, M. Callisti, T. Polcar, Z. Sofer, Chem. Eur. J. 23, 8082 (2017).

[31] C-Li. Lo, M. Catalano, A. Khosravi, W. Ge, Y. Ji, D.Y. Zemlyanov, L. Wang et al., Adv. Mater. 31, 1902397 (2019).

[32] A.H. Soeriyadi, Y. Zhu, P. Reece, J.J. Gooding, AIP Conf. Proc. 1586, 35 (2014). 